\title{
Regio- and Enantioselective Allylic Amination of Achiral Allylic Esters Catalyzed by An Iridium-Phosphoramidite Complex
}

Toshimichi Ohmura and John F. Hartwig*

Department of Chemistry, Yale University, P.O. Box 208107, New Haven, Connecticut 065208107

\section{Supporting Information}

General Procedures. ${ }^{1} \mathrm{H}$ NMR spectra were recorded at $400.13 \mathrm{MHz}$ with $\mathrm{CDCl}_{3}$ as the solvent and tetramethylsilane as the internal standard. ${ }^{13} \mathrm{C}\left\{{ }^{1} \mathrm{H}\right\}$ NMR spectra were obtained at 100.59 MHz or $125.77 \mathrm{MHz}$ with $\mathrm{CDCl}_{3}$ as the solvent. Chemical shifts of the ${ }^{13} \mathrm{C}$ NMR spectra were measured relative to $\mathrm{CDCl}_{3}(77.0 \mathrm{ppm})$. Optical rotations were measured with in a $10 \mathrm{~cm}$ cell (concentration $c$ given as $\mathrm{g} / 100 \mathrm{~mL}$ ). Elemental Analyses were performed by Robertson Microlit Laboratories, Inc., Madison, NJ 07940.

All reactions were conducted using standard Schlenk and drybox techniques. THF, $\mathrm{Et}_{2} \mathrm{O}$, and toluene were distilled from sodium-benzophenone ketyl under nitrogen. All other solvents were purchased as anhydrous grade reagents and used without further purification. $\left[\operatorname{Ir}(\operatorname{cod}) \mathrm{Cl}_{2}{ }_{2}{ }^{1}\right.$, $O, O^{\prime}$-(R)-(1,1'-Dinaphthyl-2,2'-diyl)- $N, N^{\prime}$-di- $(R, R)$-1-phenylethylphosphoramidite ${ }^{2} \quad\left[\left(R_{a}, R_{C}, R_{C}\right)\right.$ $6 \quad$ ], $O, O^{\prime}-(S)-\left(1,1^{\prime}\right.$-Dinaphthyl-2,2'-diyl)- $N, N^{\prime}$-di- $(R, R)-1$-phenylethylphosphoramidite ${ }^{2}$ $\left[\left(S_{a}, R_{C}, R_{C}\right)\right.$-6 $], O, O^{\prime}-(R)-\left(1,1^{\prime}\right.$-Dinaphthyl-2,2'-diyl)- $N, N^{\prime}$-diisopropylphosphoramidite ${ }^{2}[(R)-8]$, and $O, O^{\prime}$-(1,1'-Diphenyl-2,2'-diyl)- $N, N^{\prime}$-di- $(R, R)$-1-phenylethylphosphoramidite ${ }^{2}[(R, R)-9]$ were prepared according to published procedures. $O, O^{\prime}-(R)-\left(1,1^{\prime}\right.$-Dinaphthyl-2,2'-diyl)- $N, N^{\prime}$ dimethylphosphoramidite $[(R)-7]$ was purchased from Strem Chemicals. All allylic carbonates (1a - 1f) were synthesized by the reaction of corresponding allylic alcohols with methyl chloroformate in the presence of pyridine. (E)-4-Methoxycinnamyl alcohol, (E)-2methoxycinnamyl alcohol, and (E)-3-(2-furanyl)-2-propen-1-ol were prepared by the DIBAH reduction of corresponding aldehydes. All amines $(\mathbf{2 a}-\mathbf{2 h}),(E)$-cinnamyl alcohol, $(E)$-2-hexen1-ol, 2-methoxycinnamaldehyde, (E)-3-(2-furyl)acrolein, cinnamyl acetate (1g) (Aldrich Chemicals Co.), (E)-4-methoxycinnamaldehyde (TCI), and 4-nitrocinnamyl alcohol (Alfa Aesar) were purchased and used without further purification.

General Procedure for the Enantioselective Allylic Amination Catalyzed by Iridium-Phosphoramidite Complex: A typical procedure is given for the reaction of entry 1 in Table 1 . In a drybox, $[\mathrm{Ir}(\mathrm{cod}) \mathrm{Cl}]_{2}(6.5 \mathrm{mg}, 0.010 \mathrm{mmol})$ and $\left(R_{a}, R_{C}, R_{C}\right)-6(10.8 \mathrm{mg}, 0.0200$ $\mathrm{mmol}$ ) were dissolved in $0.5 \mathrm{~mL}$ of THF in a screw-capped vial. A small magnetic stirbar was added, and the vial was sealed with a cap containing a PTFE septum and removed from the 
drybox. Benzylamine (2a, $135 \mathrm{mg}, 1.26 \mathrm{mmol}$ ) and cinnamyl methycarbonate (1a, $188 \mathrm{mg}$, $0.979 \mathrm{mmol}$ ) were added to the reaction mixture by syringe. The reaction mixture was stirred at room temperature for $10 \mathrm{~h}$ and monitored by GC. After the reaction was complete, the volatile materials were evaporated. ${ }^{1} \mathrm{H}$ NMR analysis of the residual crude mixture indicated the ratio of regioisomers 3/4 and diallylation product 5 to be 98/1/1. The mixture was then purified by flash column chromatography on silica gel (2.5\% ethyl acetate in hexane) to give $(R)-N$-(1-phenyl-2propenyl)benzylamine (184 mg, 84\%). HPLC analysis indicated that the enantiomeric excess of product was 95\% [Daicel CHIRALCEL OD-H $(0.46 \mathrm{~cm} \times 25 \mathrm{~cm})$; hexane/2propanol/diethylamine $=99.74 / 0.25 / 0.01 ;$ flow rate $=0.6 \mathrm{~mL} / \mathrm{min}$; detection wave length $=220$ $\mathrm{nm} ; \mathrm{T}_{\mathrm{R}}=15.1$ (major), 17.4 (minor) min].

(R)- $N$-(1-Phenyl-2-propenyl)benzylamine: ${ }^{3}{ }^{1} \mathrm{H} \quad \mathrm{NMR}\left(400.13 \mathrm{MHz}, \mathrm{CDCl}_{3}\right) \quad \delta$ $7.30-7.39(\mathrm{~m}, 8 \mathrm{H}), 7.22-7.28(\mathrm{~m}, 2 \mathrm{H}), 5.95$ (ddd, $J=16.8,10.0,7.2 \mathrm{~Hz}, 1 \mathrm{H}), 5.23$ (dt, $J=16.8$, $1.6 \mathrm{~Hz}, 1 \mathrm{H}), 5.12(\mathrm{dq}, J=10.0,0.8 \mathrm{~Hz}, 1 \mathrm{H}), 4.23(\mathrm{~d}, J=7.2 \mathrm{~Hz}, 1 \mathrm{H}), 3.75(\mathrm{~d}$ of AB pattern, $J=$ $13.4 \mathrm{~Hz}, 1 \mathrm{H}), 3.71$ (d of AB pattern, $J=13.4 \mathrm{~Hz}, 1 \mathrm{H}), 1.64$ (brs, $1 \mathrm{H}) .{ }^{13} \mathrm{C}$ NMR $(100.59 \mathrm{MHz}$, $\left.\mathrm{CDCl}_{3}\right) \delta 142.74,140.90,140.38,128.53,128.37,128.15,127.31,127.21,126.89,115.18,65.09$, 51.26. The absolute configuration was determined by comparison of the optical rotation with literature data: $[\alpha]_{\mathrm{D}}^{\mathrm{RT}}=-7.0\left(c 3.1, \mathrm{CHCl}_{3}\right)$, lit. $^{4}[\alpha]_{\mathrm{D}}{ }^{25}=+3.6\left(c 0.5, \mathrm{CHCl}_{3}\right)$ for $(S)$-enantiomer of $86 \%$ ee.

$N$-(1-Phenyl-2-propenyl)-4-methoxybenzylamine 4 (Table 2, entry 1): The general procedure was followed with 4-methoxybenzylamine (2b, $186 \mathrm{mg}, 1.35 \mathrm{mmol})$ and $\mathbf{1 a}(199 \mathrm{mg}$, $1.04 \mathrm{mmol}$ ). The reaction was conducted at room temperature for $18 \mathrm{~h} .{ }^{1} \mathrm{H}$ NMR analysis of the crude mixture indicated the ratio of regioisomers $3 / 4$ and diallylation product 5 to be 98/0/2. The mixture was purified by flash column chromatography on silica gel (5\% ethyl acetate in hexane) to give the title compound ( $210 \mathrm{mg}, 80 \%)$. HPLC analysis indicated that the enantiomeric excess of product was $94 \%$ [Daicel CHIRALCEL OD-H $(0.46 \mathrm{~cm} \times 25 \mathrm{~cm})$; hexane $/ 2-$ propanol = 99.75/0.25; flow rate $=0.5 \mathrm{~mL} / \mathrm{min}$; detection wave length $=230 \mathrm{~nm} ; \mathrm{T}_{\mathrm{R}}=26.2$ (major), 29.3 (minor) min]: $[\alpha]_{\mathrm{D}}{ }^{\mathrm{RT}}=-1.8\left(c 5.5, \mathrm{CHCl}_{3}\right) .{ }^{1} \mathrm{H} \mathrm{NMR}\left(400.13 \mathrm{MHz}, \mathrm{CDCl}_{3}\right) \delta$ 7.21-7.38 (m, 7H), $6.86(\mathrm{~d}, J=8.4 \mathrm{~Hz}, 2 \mathrm{H}), 5.94(\mathrm{ddd}, J=17.2,10.0,7.6 \mathrm{~Hz}, 1 \mathrm{H}), 5.22(\mathrm{dt}, J=17.2,1.4 \mathrm{~Hz}, 1 \mathrm{H})$, 5.11 (ddd, $J=10.0,1.6,1.2 \mathrm{~Hz}, 1 \mathrm{H}), 4.21(\mathrm{~d}, J=7.6 \mathrm{~Hz}, 1 \mathrm{H}), 3.80(\mathrm{~s}, 3 \mathrm{H}), 3.68(\mathrm{~d}$ of AB pattern, $J=13.0 \mathrm{~Hz}, 1 \mathrm{H}$ ), 3.64 (d of AB pattern, $J=13.0 \mathrm{~Hz}, 1 \mathrm{H}$ ), 1.58 (brs, $1 \mathrm{H}$ ). ${ }^{13} \mathrm{C}$ NMR $\left(125.77 \mathrm{MHz}, \mathrm{CDCl}_{3}\right) \delta$ 158.54, 142.84, 141.01, 132.56, 129.29, 128.49, 127.30, 127.14, 115.05, $113.72,64.99,55.24,50.65$.

(R)- $N$-(1-Phenyl-2-propenyl)-n-hexylamine ${ }^{5}$ (Table 2, entry 2): The general procedure was followed with $n$-hexylamine (2c, $129 \mathrm{mg}, 1.27 \mathrm{mmol})$ and $\mathbf{1 a}(193 \mathrm{mg}, 1.00 \mathrm{mmol})$. The reaction was conducted at room temperature for $9 \mathrm{~h} .{ }^{1} \mathrm{H}$ NMR analysis of the crude mixture indicated the ratio of regioisomers $\mathbf{3 / 4}$ and diallylation product $\mathbf{5}$ to be 98/2/0. The mixture was 
purified by flash column chromatography on silica gel ( $2.5 \%$ ethyl acetate in hexane) to give the title compound (192 mg, 88\%). The HPLC analysis indicated that the enantiomeric excess of product was $96 \%$ [Daicel CHIRALCEL OD-H $(0.46 \mathrm{~cm}$ x $25 \mathrm{~cm})$; hexane/diethylamine = 99.98/0.02; flow rate $=0.4 \mathrm{~mL} / \mathrm{min}$; detection wave length $=210 \mathrm{~nm} ; \mathrm{T}_{\mathrm{R}}=19.8$ (major), 20.9 (minor) min]. The absolute configuration was determined by comparison of the optical rotation with literature data: $[\alpha]_{\mathrm{D}}{ }^{\mathrm{RT}}=-19\left(c 1.1, \mathrm{CHCl}_{3}\right)$, lit. $^{4}[\alpha]_{\mathrm{D}}{ }^{25}=+18.5\left(c 0.5, \mathrm{CHCl}_{3}\right)$ for $(S)$ enantiomer of $90 \%$ ee. ${ }^{1} \mathrm{H}$ NMR $\left(400.13 \mathrm{MHz}, \mathrm{CDCl}_{3}\right) \delta$ 7.30-7.35 (m, 4H), 7.21-7.27 (m, 1H), $5.93(\mathrm{ddd}, J=17.2,10.0,6.8 \mathrm{~Hz}, 1 \mathrm{H}), 5.20(\mathrm{dt}, J=17.2,1.4 \mathrm{~Hz}, 1 \mathrm{H}), 5.08$ (ddd, $J=10.0,1.6$, $1.2 \mathrm{~Hz}, 1 \mathrm{H}), 4.17(\mathrm{~d}, J=6.8 \mathrm{~Hz}, 1 \mathrm{H}), 2.58(\mathrm{dt}, J=11.2,7.2 \mathrm{~Hz}, 1 \mathrm{H}), 2.48(\mathrm{dt}, J=11.2,7.2 \mathrm{~Hz}$, $1 \mathrm{H}), 1.20-1.53(\mathrm{~m}, 9 \mathrm{H}), 0.87(\mathrm{t}, J=6.8 \mathrm{~Hz}, 3 \mathrm{H}) .{ }^{13} \mathrm{C} \mathrm{NMR}\left(100.59 \mathrm{MHz}, \mathrm{CDCl}_{3}\right) \delta 143.07$, 141.23, 128.46, 127.17, 127.07, 114.77, 66.28, 47.72, 31.77, 30.14, 27.04, 22.61, 14.06.

$\mathrm{N}$-(1-Phenyl-2-propenyl)allylamine (Table 2, entry 3): The general procedure was followed with allyl amine $(\mathbf{2 d}, 75.5 \mathrm{mg}, 1.32 \mathrm{mmol})$ and $\mathbf{1 a}(204 \mathrm{mg}, 1.06 \mathrm{mmol})$. The reaction was conducted at room temperature for $12 \mathrm{~h}$. The mixture was purified by flash column chromatography on silica gel (10\% ethyl acetate in hexane) to give the title compound (140 $\mathrm{mg}$, $76 \%$ ). The HPLC analysis indicated that the enantiomeric excess of product was $97 \%$ [Daicel CHIRALCEL OD-H $(0.46 \mathrm{~cm}$ x $25 \mathrm{~cm})$; hexane/diethylamine $=99.99 / 0.01$; flow rate $=0.5$ $\mathrm{mL} / \mathrm{min}$; detection wave length $=210 \mathrm{~nm} ; \mathrm{T}_{\mathrm{R}}=17.9$ (major), 19.2 (minor) min]: $[\alpha]_{\mathrm{D}}{ }^{\mathrm{RT}}=-14(c$ 3.1, $\left.\mathrm{CHCl}_{3}\right)$. ${ }^{1} \mathrm{H} \mathrm{NMR}\left(400.13 \mathrm{MHz}, \mathrm{CDCl}_{3}\right) \delta$ 7.30-7.35 (m, 4H), 7.22-7.28 (m, 1H), 5.83-5.88 $(\mathrm{m}, 2 \mathrm{H}), 5.21(\mathrm{dt}, J=17.2,1.2 \mathrm{~Hz}, 1 \mathrm{H}), 5.16(\mathrm{dq}, J=17.2,1.6 \mathrm{~Hz}, 1 \mathrm{H}), 5.11(\mathrm{dt}, J=10.0,1.2$ $\mathrm{Hz}, 1 \mathrm{H}), 5.09(\mathrm{dq}, J=10.0,1.6 \mathrm{~Hz}, 1 \mathrm{H}), 4.23(\mathrm{~d}, J=7.2 \mathrm{~Hz}, 1 \mathrm{H}), 3.21$ (ddt of AB pattern, $J=$ 14.2, 6.0, $1.4 \mathrm{~Hz}, 1 \mathrm{H}$ ), 3.16 (ddt of AB pattern, $J=14.2,6.0,1.4 \mathrm{~Hz}, 1 \mathrm{H}$ ), 1.41 (brs, $1 \mathrm{H}$ ). ${ }^{13} \mathrm{C}$ NMR (100.59 MHz, $\left.\mathrm{CDCl}_{3}\right) \delta 142.69,140.82,136.72,128.50,127.24,127.18,115.90,115.09$, 65.17, 49.88. Anal. Calcd for $\mathrm{C}_{12} \mathrm{H}_{15} \mathrm{~N}$ : C, 83.19; H, 8.73; N, 8.08. Found: C, 83.06; H, 8.61; N, 8.06 .

1-(1-Phenyl-2-propenyl)pyrrolidine ${ }^{6}$ (Table 2, entry 4): The general procedure was followed with pyrrolidine $(\mathbf{2 e}, 86.9 \mathrm{mg}, 1.22 \mathrm{mmol})$ and $\mathbf{1 a}(185 \mathrm{mg}, 0.96 \mathrm{mmol})$. The reaction was conducted at room temperature for $2 \mathrm{~h} .{ }^{1} \mathrm{H} \mathrm{NMR}$ analysis of the crude mixture indicated the ratio of regioisomers $\mathbf{3 / 4}$ to be $98 / 2$. The mixture was purified by flash column chromatography on silica gel (5\% ethyl acetate in hexane) to give the title compound (135 $\mathrm{mg}, 75 \%$ ). HPLC analysis indicated that the enantiomeric excess of product was 97\% [Daicel CHIRALCEL OD-H $(0.46 \mathrm{~cm} \times 25 \mathrm{~cm})$; hexane $/$ diethylamine $=99.99 / 0.01$; flow rate $=0.6 \mathrm{~mL} / \mathrm{min}$; detection wave length $=210 \mathrm{~nm} ; \mathrm{T}_{\mathrm{R}}=11.2$ (major), 12.5 (minor) min]: $[\alpha]_{\mathrm{D}}{ }^{\mathrm{RT}}=-85\left(c 3.2, \mathrm{CHCl}_{3}\right) .{ }^{1} \mathrm{H} \mathrm{NMR}$

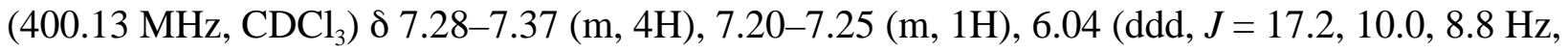
$1 \mathrm{H}), 5.20(\mathrm{dd}, J=17.2,1.2 \mathrm{~Hz}, 1 \mathrm{H}), 5.00(\mathrm{dd}, J=10.0,1.2 \mathrm{~Hz}, 1 \mathrm{H}), 3.58(\mathrm{~d}, J=8.8 \mathrm{~Hz}, 1 \mathrm{H})$, 
2.45-2.54 (m, 2H), 2.33-2.42 (m, 2H), $1.76(\mathrm{~m}, 4 \mathrm{H}) .{ }^{13} \mathrm{C} \mathrm{NMR}\left(100.59 \mathrm{MHz}, \mathrm{CDCl}_{3}\right) \delta 142.73$, $141.02,128.48,127.57,127.12,115.09,75.20,52.97,23.27$.

1-(1-Phenyl-2-propenyl)piperidine ${ }^{7}$ (Table 2, entry 5): The general procedure was followed with piperidine (2f, $114 \mathrm{mg}, 1.33 \mathrm{mmol})$ and $\mathbf{1 a}(194 \mathrm{mg}, 1.01 \mathrm{mmol})$. The reaction was conducted at room temperature for $10 \mathrm{~h} .{ }^{1} \mathrm{H}$ NMR analysis of the crude mixture indicated the ratio of regioisomers $\mathbf{3 / 4}$ to be $97 / 3$. The mixture was purified by flash column chromatography on silica gel (2.5\% ethyl acetate in hexane) to give the title compound (185 mg, 91\%). HPLC analysis indicated that the enantiomeric excess of product was $96 \%$ [Daicel CHIRALCEL OJ $(0.46 \mathrm{~cm} \times 25 \mathrm{~cm})$; hexane $/ 2$-propanol $/$ diethylamine $=99.97 / 0.02 / 0.01 ;$ flow rate $=0.6 \mathrm{~mL} / \mathrm{min}$; detection wave length $=210 \mathrm{~nm} ; \mathrm{T}_{\mathrm{R}}=9.3$ (minor), 13.1 (major) min $]:[\alpha]_{\mathrm{D}}{ }^{\mathrm{RT}}=-99$ (c 3.2, $\mathrm{CHCl}_{3}$ ). ${ }^{1} \mathrm{H}$ NMR $\left(400.13 \mathrm{MHz}, \mathrm{CDCl}_{3}\right) \delta$ 7.28-7.35 (m, 4H), 7.20-7.24 (m, 1H), 5.94 (ddd, $J=$ $17.2,10.4,8.5 \mathrm{~Hz}, 1 \mathrm{H}), 5.18(\mathrm{dd}, J=17.2,1.6 \mathrm{~Hz}, 1 \mathrm{H}), 5.07(\mathrm{dd}, J=10.4,1.6 \mathrm{~Hz}, 1 \mathrm{H}), 3.65(\mathrm{~d}$, $J=8.5 \mathrm{~Hz}, 1 \mathrm{H}), 2.35-2.48(\mathrm{~m}, 2 \mathrm{H}), 2.22-2.34(\mathrm{~m}, 2 \mathrm{H}), 1.50-1.59(\mathrm{~m}, 4 \mathrm{H}), 1.36-1.45(\mathrm{~m}, 2 \mathrm{H})$. ${ }^{13} \mathrm{C}$ NMR $\left(125.77 \mathrm{MHz}, \mathrm{CDCl}_{3}\right) \delta 142.41,140.39,128.34,127.95,126.87,115.82,75.42,52.46$, $26.15,24.65$.

1-(1-Phenyl-2-propenyl)morpholine ${ }^{8}$ (Table 2, entry 6): The general procedure was followed with morpholine $(\mathbf{2 g}, 113 \mathrm{mg}, 1.30 \mathrm{mmol})$ and $\mathbf{1 a}(187 \mathrm{mg}, 0.974 \mathrm{mmol})$. The reaction was conducted at room temperature for $24 \mathrm{~h} .{ }^{1} \mathrm{H}$ NMR analysis of the crude mixture indicated the ratio of regioisomers $\mathbf{3 / 4}$ to be $99 / 1$. The mixture was purified by flash column chromatography on silica gel (2.5\% ethyl acetate in hexane) to give the title compound (183 $\mathrm{mg}, 92 \%)$. HPLC analysis indicated that the enantiomeric excess of product was 97\% [Daicel CHIRALCEL OJ $(0.46 \mathrm{~cm} \times 25 \mathrm{~cm})$; hexane $/ 2$-propanol $/$ diethylamine $=98.99 / 1 / 0.01 ;$ flow rate $=0.5 \mathrm{~mL} / \mathrm{min}$; detection wave length $=210 \mathrm{~nm} ; \mathrm{T}_{\mathrm{R}}=12.4$ (minor), 13.7 (major) min $]:[\alpha]_{\mathrm{D}}^{\mathrm{RT}}=-98(c 5.2$, $\mathrm{CHCl}_{3}$ ). ${ }^{1} \mathrm{H}$ NMR $\left(400.13 \mathrm{MHz}, \mathrm{CDCl}_{3}\right) \delta$ 7.29-7.36 (m, 4H), 7.22-7.26 (m, 1H), 5.90 (ddd, $J=$ $17.2,10.0,8.8 \mathrm{~Hz}, 1 \mathrm{H}), 5.23(\mathrm{dd}, J=17.2,1.6 \mathrm{~Hz}, 1 \mathrm{H}), 5.10(\mathrm{dd}, J=10.0,1.6 \mathrm{~Hz}, 1 \mathrm{H}), 3.69$ (t, $J$ $=4.0 \mathrm{~Hz}, 4 \mathrm{H}), 3.62(\mathrm{~d}, J=8.8 \mathrm{~Hz}, 1 \mathrm{H}), 2.43-2.55(\mathrm{~m}, 2 \mathrm{H}), 2.29-2.37(\mathrm{~m}, 2 \mathrm{H}) .{ }^{13} \mathrm{C}$ NMR $\left(100.59 \mathrm{MHz}, \mathrm{CDCl}_{3}\right) \delta$ 141.54, 139.70, 128.57, 127.92, 127.24, 116.64, 75.51, 67.12, 51.96.

(1-Phenyl-2-propenyl)diethylamine ${ }^{3}$ (Table 2, entry 7): The general procedure was followed with diethylamine $(\mathbf{2 h}, 91.1 \mathrm{mg}, 1.25 \mathrm{mmol})$ and $\mathbf{1 a}(193 \mathrm{mg}, 1.01 \mathrm{mmol})$. The reaction was conducted at $50{ }^{\circ} \mathrm{C}$ for $16 \mathrm{~h} .{ }^{1} \mathrm{H}$ NMR analysis of the crude mixture indicated the ratio of regioisomer $\mathbf{3 / 4}$ to be $98 / 2$. The mixture was purified by flash column chromatography on silica gel (2.5\% ethyl acetate in hexane) to give the title compound $(158 \mathrm{mg}, 83 \%)$. HPLC analysis indicated that the enantiomeric excess of product was 97\% [Daicel CHIRALCEL OJ $(0.46 \mathrm{~cm} \mathrm{x}$ $25 \mathrm{~cm}$ ); hexane $/$ diethylamine $=99.99 / 0.01$; flow rate $=0.4 \mathrm{~mL} / \mathrm{min}$; detection wave length $=210$ $\mathrm{nm} ; \mathrm{T}_{\mathrm{R}}=12.6$ (minor), 13.6 (major) min]: $[\alpha]_{\mathrm{D}}{ }^{\mathrm{RT}}=-106\left(c 3.1, \mathrm{CHCl}_{3}\right) .{ }^{1} \mathrm{H} \mathrm{NMR}(400.13 \mathrm{MHz}$, $\left.\mathrm{CDCl}_{3}\right) \delta 7.19-7.40(\mathrm{~m}, 5 \mathrm{H}), 5.95$ (ddd, $\left.J=17.2,10.0,8.8 \mathrm{~Hz}, 1 \mathrm{H}\right), 5.19$ (dd, $J=17.2,1.6 \mathrm{~Hz}$, 
$1 \mathrm{H}), 5.12(\mathrm{dd}, J=10.0,1.6 \mathrm{~Hz}, 1 \mathrm{H}), 4.13(\mathrm{~d}, J=8.8 \mathrm{~Hz}, 1 \mathrm{H}), 2.49-2.63(\mathrm{~m}, 4 \mathrm{H}), 0.98(\mathrm{t}, J=7.2$ $\mathrm{Hz}, 6 \mathrm{H}) .{ }^{13} \mathrm{C}$ NMR $\left(100.59 \mathrm{MHz}, \mathrm{CDCl}_{3}\right) \delta 142.96,139.50,128.29,127.82,126.79,116.06$, $69.46,42.82,11.44$.

$N$-[1-(4-Methoxyphenyl)-2-propenyl]benzylamine ${ }^{9}$ (Table 2, entry 8): The general procedure was followed with $\mathbf{2 a}(129 \mathrm{mg}, 1.20 \mathrm{mmol})$ and 4-methoxycinnamyl methylcarbonate (1b, $222 \mathrm{mg}, 1.00 \mathrm{mmol})$. The reaction was conducted at room temperature for $9 \mathrm{~h} .{ }^{1} \mathrm{H}$ NMR analysis of the crude mixture indicated the ratio of regioisomers $3 / 4$ and diallylation product 5 to be $99 / 1 / 0$. The mixture was purified by flash column chromatography on silica gel (5\% ethyl acetate in hexane) to give the title compound ( $223 \mathrm{mg}$, 88\%). HPLC analysis indicated that the enantiomeric excess of product was 96\% [Daicel CHIRALCEL OD-H $(0.46 \mathrm{~cm} \times 25 \mathrm{~cm})$; hexane $/ 2$-propanol $=98 / 2$; flow rate $=0.6 \mathrm{~mL} / \mathrm{min}$; detection wave length $=230 \mathrm{~nm} ; \mathrm{T}_{\mathrm{R}}=10.5$ (major), 12.0 (minor) min]: $[\alpha]_{\mathrm{D}}^{\mathrm{RT}}=-0.43\left(\right.$ c 5.1, $\left.\mathrm{CHCl}_{3}\right) .{ }^{1} \mathrm{H}$ NMR $\left(400.13 \mathrm{MHz}, \mathrm{CDCl}_{3}\right) \delta$ $7.21-7.33(\mathrm{~m}, 7 \mathrm{H}), 6.88(\mathrm{~d}, J=8.8 \mathrm{~Hz}, 2 \mathrm{H}), 5.93(\mathrm{ddd}, J=17.2,10.0,7.6 \mathrm{~Hz}, 1 \mathrm{H}), 5.20(\mathrm{dt}, J=$ $17.2,1.6 \mathrm{~Hz}, 1 \mathrm{H}), 5.10(\mathrm{dt}, J=10.0,1.6 \mathrm{~Hz}, 1 \mathrm{H}), 4.18(\mathrm{~d}, J=7.6 \mathrm{~Hz}, 1 \mathrm{H}), 3.80(\mathrm{~s}, 3 \mathrm{H}), 3.74(\mathrm{~d}$ of AB pattern, $J=13.4 \mathrm{~Hz}, 1 \mathrm{H}$ ), 3.70 (d of AB pattern, $J=13.4 \mathrm{~Hz}, 1 \mathrm{H}$ ), 1.59 (brs, $1 \mathrm{H}$ ). ${ }^{13} \mathrm{C}$ NMR $\left(100.59 \mathrm{MHz}, \mathrm{CDCl}_{3}\right) \delta 158.71,141.12,140.42,134.84,128.35,128.33,128.13,126.85$, $114.84,113.86,64.39,55.24,51.19$.

$N$-[1-(4-Nitrophenyl)-2-propenyl]benzylamine (Table 2, entry 9): The general procedure was followed with $[\operatorname{Ir}(\mathrm{cod}) \mathrm{Cl}]_{2}(3.3 \mathrm{mg}, 0.0050 \mathrm{mmol}),\left(R_{a}, R_{C}, R_{C}\right)-6(5.4 \mathrm{mg}, 0.010$ $\mathrm{mmol})$, THF $(0.25 \mathrm{~mL}), \mathbf{2 a}(111 \mathrm{mg}, 1.03 \mathrm{mmol})$ and 4-nitrocinnamyl methylcarbonate (1c, 103 $\mathrm{mg}, 0.43 \mathrm{mmol})$. The reaction was conducted at room temperature for $12 \mathrm{~h} .{ }^{1} \mathrm{H}$ NMR analysis of the crude mixture indicated the ratio of regioisomers $3 / 4$ and diallylation product 5 to be 83/13/4. The mixture was purified by flash column chromatography on silica gel $\left(50 \% \mathrm{CH}_{2} \mathrm{Cl}_{2}\right.$ in hexane) to give the title compound $(78.1 \mathrm{mg}, 67 \%)$. HPLC analysis indicated that the enantiomeric excess of product was $86 \%$ [Daicel CHIRALCEL OD-H $(0.46 \mathrm{~cm} \times 25 \mathrm{~cm})$; hexane/2propanol $/$ diethylamine $=99.4 / 0.5 / 0.1$; flow rate $=0.6 \mathrm{~mL} / \mathrm{min}$; detection wave length $=270 \mathrm{~nm}$; $\mathrm{T}_{\mathrm{R}}=45.2$ (minor), 51.1 (major) min]: $[\alpha]_{\mathrm{D}}^{\mathrm{RT}}=-12\left(c\right.$ 0.74, $\left.\mathrm{CHCl}_{3}\right) .{ }^{1} \mathrm{H}$ NMR $(400.13 \mathrm{MHz}$, $\left.\mathrm{CDCl}_{3}\right) \delta 8.19(\mathrm{~d}, J=7.2 \mathrm{~Hz}, 2 \mathrm{H}), 7.57(\mathrm{~d}, J=7.2 \mathrm{~Hz}, 2 \mathrm{H}), 7.24-7.35(\mathrm{~m}, 5 \mathrm{H}), 5.87$ (ddd, $J=$ $17.2,10.4,7.2 \mathrm{~Hz}, 1 \mathrm{H}), 5.26(\mathrm{~d}, J=17.2 \mathrm{~Hz}, 1 \mathrm{H}), 5.19(\mathrm{~d}, J=10.4 \mathrm{~Hz}, 1 \mathrm{H}), 4.33(\mathrm{~d}, J=7.2 \mathrm{~Hz}$, $1 \mathrm{H}$ ), $3.74(\mathrm{~d}$ of AB pattern, $J=13.2 \mathrm{~Hz}, 1 \mathrm{H}$ ), 3.69 (d of AB pattern, $J=13.2,1 \mathrm{H}), 1.67$ (brs, 1H). ${ }^{13} \mathrm{C}$ NMR $\left(125.77 \mathrm{MHz}, \mathrm{CDCl}_{3}\right) \delta 150.35,147.14,139.77,139.51,128.45,128.14,128.06$, 127.12, 123.74, 116.56, 64.58, 51.26. Anal. Calcd for $\mathrm{C}_{16} \mathrm{H}_{16} \mathrm{~N}_{2} \mathrm{O}_{2}: \mathrm{C}, 71.62 ; \mathrm{H}, 6.01 ; \mathrm{N}, 10.44$. Found: C, 71.39; H, 5.99; N, 10.31 .

$N$-[1-(2-Methoxyphenyl)-2-propenyl]benzylamine (Table 2, entry 10): The general procedure was followed with $\mathbf{2 a}(139 \mathrm{mg}, 1.30 \mathrm{mmol})$ and 2-methoxycinnamil methylcarbonate (1d, $254 \mathrm{mg}, 1.00 \mathrm{mmol})$. The reaction was conducted at room temperature for $16 \mathrm{~h}$. ${ }^{1} \mathrm{H}$ NMR 
analysis of the crude mixture indicated the ratio of regioisomers $3 / 4$ and diallylation product 5 to be $95 / 4 / 1$. The mixture was purified by flash column chromatography on silica gel (5\% ethyl acetate in hexane) to give the title compound (196 mg, 77\%). HPLC analysis indicated that the enantiomeric excess of product was 76\% [Daicel CHIRALCEL OD-H $(0.46 \mathrm{~cm} \times 25 \mathrm{~cm})$; hexane $/ 2$-propanol/diethylamine $=99.49 / 0.5 / 0.01$; flow rate $=0.5 \mathrm{~mL} / \mathrm{min}$; detection wave length $=220 \mathrm{~nm} ; \mathrm{T}_{\mathrm{R}}=23.3$ (major), 25.1 (minor) min $]:[\alpha]_{\mathrm{D}}{ }^{\mathrm{RT}}=-4.6\left(\right.$ c 5.2, $\left.\mathrm{CHCl}_{3}\right) .{ }^{1} \mathrm{H} \mathrm{NMR}$ $\left(400.13 \mathrm{MHz}, \mathrm{CDCl}_{3}\right) \delta 7.28-7.36(\mathrm{~m}, 5 \mathrm{H}), 7.21-7.26(\mathrm{~m}, 2 \mathrm{H}), 6.96(\mathrm{dt}, J=7.6,0.8 \mathrm{~Hz}, 1 \mathrm{H})$, $6.88(\mathrm{dd}, J=8.4,0.8 \mathrm{~Hz}, 1 \mathrm{H}), 6.03(\mathrm{ddd}, J=17.2,10.4,6.8 \mathrm{~Hz}, 1 \mathrm{H}), 5.21(\mathrm{dt}, J=17.2,1.6 \mathrm{~Hz}$, $1 \mathrm{H}), 5.11$ (ddd, $J=10.4,1.6,1.0 \mathrm{~Hz}, 1 \mathrm{H}), 4.58(\mathrm{~d}, J=6.8 \mathrm{~Hz}, 1 \mathrm{H}), 3.81(\mathrm{~s}, 3 \mathrm{H}), 3.74(\mathrm{~d}$ of AB pattern, $J=13.2,1 \mathrm{H}$ ), 3.69 (d of AB pattern, $J=13.2 \mathrm{~Hz}, 1 \mathrm{H}$ ), 1.83 (brs, $1 \mathrm{H}$ ). ${ }^{13} \mathrm{C}$ NMR $(100.59$ $\left.\mathrm{MHz}, \mathrm{CDCl}_{3}\right) \delta 157.10,140.63,139.90,130.66,128.25,128.22,128.10,128.05,126.74,120.78$, 114.95, 110.71, 59.57, 55.32, 51.47. Anal. Calcd for $\mathrm{C}_{17} \mathrm{H}_{19} \mathrm{NO}$ : C, 80.60; H, 7.56; N, 5.53. Found: C, 80.68; H, 7.55; N, 5.72 .

$N$-[1-(2-Furyl)-2-propenyl]benzylamine ${ }^{10}$ (Table 2, entry 11): The general procedure was followed with $\mathbf{2 a}(130 \mathrm{mg}, 1.21 \mathrm{mmol})$ and 3-(2-furanyl)-2-propenyl methylcarbonate (1e, $182 \mathrm{mg}, 1.00 \mathrm{mmol})$. The reaction was conducted at room temperature for $10 \mathrm{~h} .{ }^{1} \mathrm{H}$ NMR analysis of the crude mixture indicated the ratio of regioisomers $3 / 4$ and diallylation product 5 to be $96 / 2 / 2$. The mixture was purified by flash column chromatography on silica gel ( $2.5 \%$ ethyl acetate in hexane) to give the title compound (125 mg, 58\%). HPLC analysis indicated that the enantiomeric excess of product was 97\% [Daicel CHIRALCEL OD-H $(0.46 \mathrm{~cm} \times 25 \mathrm{~cm})$; hexane $/ 2$-propanol $=98 / 2 ;$ flow rate $=0.5 \mathrm{~mL} / \mathrm{min}$; detection wave length $=230 \mathrm{~nm} ; \mathrm{T}_{\mathrm{R}}=10.3$ (major), 11.2 (minor) min]: $[\alpha]_{\mathrm{D}}^{\mathrm{RT}}=+9.0\left(c 3.0, \mathrm{CHCl}_{3}\right) .{ }^{1} \mathrm{H} \mathrm{NMR}\left(400.13 \mathrm{MHz}, \mathrm{CDCl}_{3}\right) \delta 7.38$ $(\mathrm{dd}, J=1.8,0.6 \mathrm{~Hz}, 1 \mathrm{H}), 7.29-7.34(\mathrm{~m}, 4 \mathrm{H}), 7.22-7.28(\mathrm{~m}, 1 \mathrm{H}), 6.33(\mathrm{dd}, J=3.2,1.8 \mathrm{~Hz}, 1 \mathrm{H})$, $6.20(\mathrm{~d}, J=3.2 \mathrm{~Hz}, 1 \mathrm{H}), 5.98(\mathrm{ddd}, J=17.2,10.0,7.2 \mathrm{~Hz}, 1 \mathrm{H}), 5.26(\mathrm{dt}, J=17.2,1.2 \mathrm{~Hz}, 1 \mathrm{H})$, $5.23(\mathrm{dt}, J=10.0,1.2 \mathrm{~Hz}, 1 \mathrm{H}), 4,31(\mathrm{~d}, J=7.2 \mathrm{~Hz}, 1 \mathrm{H}), 3.77(\mathrm{~s}, 2 \mathrm{H}), 1.68$ (brs, $1 \mathrm{H}) .{ }^{13} \mathrm{C}$ NMR $\left(125.77 \mathrm{MHz}, \mathrm{CDCl}_{3}\right) \delta 155.31,141.82,140.01,137.50,128.39,128.22,126.97,116.87,110.08$, 106.37, 58.31, 50.98.

$N$-[1-(n-Propyl)-2-propenyl]benzylamine ${ }^{4}$ (Table 2, entry 12): The general procedure was followed with $\mathbf{2 a}(145 \mathrm{mg}, 1.35 \mathrm{mmol})$ and 2-hexenyl methylcarbonate (1f, $160 \mathrm{mg}, 1.01$ mmol). The reaction was conducted at room temperature for $10 \mathrm{~h} .{ }^{1} \mathrm{H}$ NMR analysis of the crude mixture indicated the ratio of regioisomers $\mathbf{3 / 4}$ and diallylation product 5 to be $88 / 8 / 4$. The mixture was purified by flash column chromatography on silica gel $(2.5 \%$ ethyl acetate in hexane) to give the title compound (127 mg, 66\%). HPLC analysis indicated that the enantiomeric excess of product was 95\% [Daicel CHIRALCEL OD-H $(0.46 \mathrm{~cm} \times 25 \mathrm{~cm})$; hexane $/ 2$-propanol $/$ diethylamine $=99.49 / 0.5 / 0,01$; flow rate $=0.6 \mathrm{~mL} / \mathrm{min}$; detection wave length $=210 \mathrm{~nm} ; \mathrm{T}_{\mathrm{R}}=8.9$ (major), 11.6 (minor) min $]:[\alpha]_{\mathrm{D}}{ }^{\mathrm{RT}}=+1.1\left(\right.$ c 5.1, $\left.\mathrm{CHCl}_{3}\right) .{ }^{1} \mathrm{H} \mathrm{NMR}$ 
$\left(400.13 \mathrm{MHz}, \mathrm{CDCl}_{3}\right) \delta 7.22-7.32(\mathrm{~m}, 5 \mathrm{H}), 5.62(\mathrm{ddd}, J=16.8,10.4,7.6 \mathrm{~Hz}, 1 \mathrm{H}), 5.14(\mathrm{dd}, J=$ $10.4,1.6 \mathrm{~Hz}, 1 \mathrm{H}), 5.10(\mathrm{dd}, J=16.8,1.6 \mathrm{~Hz}, 1 \mathrm{H}), 3.83(\mathrm{~d}, J=13.2 \mathrm{~Hz}, 1 \mathrm{H}), 3.64,(\mathrm{~d}, J=13.2$ $\mathrm{Hz}, 1 \mathrm{H}), 3.03(\mathrm{dt}, J=7.6,6.0 \mathrm{~Hz}, 1 \mathrm{H}), 1.25-1.52(\mathrm{~m}, 5 \mathrm{H}), 0.89(\mathrm{t}, J=7.2 \mathrm{~Hz}, 3 \mathrm{H}) .{ }^{13} \mathrm{C}$ NMR $\left(125.77 \mathrm{MHz}, \mathrm{CDCl}_{3}\right) \delta 141.37,140.72,128.34,128.16,126.76,115.92,61.00,51.22,37.93$, $19.08,14.06$.

\section{References}

(1) Herde, J. L.; Lambert, J. C.; Senoff, C. V. Inorg. Synth. 1974, 15, 18.

(2) Alexakis, A.; Rosset, S.; Allamand, J.; March, S.; Guillen, F.; Benhaim, C. Synlett 2001, 1375.

(3) Takeuchi, R.; Ue, N.; Tanabe, K.; Yamashita, K.; Shiga, N. J. Am. Chem. Soc. 2001, 123, 9525.

(4) Yadav, J. S.; Bandyopadhyay, A.; Reddy, B. V. S. Tetrahedron Lett. 2001, 42, 6385.

(5) Xu, Q.; Dittmer, D. C. Terahedron Lett. 1999, 40, 2255.

(6) Trost, B. M.; Spagnol, M. D. J. Chem. Soc., Perkin Trans 1 1995, 2083.

(7) Kondo, T.; Ono, H.; Satake, N.; Mitsudo, T.; Watanabe, Y. 1995, 14, 1945.

(8) Bergbreiter, D. E.; Weatherford, D. A. J. Org. Chem. 1989, 54, 2726.

(9) You, S.-L.; Zhu, X.-Z.; Luo, Y.-M.; Hou, X.-L.; Dai, L.-X. J. Am. Chem. Soc. 2001, 123, 7471.

(10) Dondoni, A.; Merchán, F. L.; Merino, P.; Tejero, T. Synth. Commun. 1994, 24, 2551. 